\title{
Interactive comment on "A framework for deriving drought indicators from GRACE" by Helena Gerdener et al.
}

\section{Anonymous Referee \#2}

Received and published: 13 September 2019

Review of HESS-2019-268 "A framework for deriving drought indicators from GRACE" by Helena Gerdener; Olga Engels; Jürgen Kusche

Recommendation- Major revision

General Comments- In this paper the authors developed a framework that potentially contributes to the understanding of how drought signals propagate through various GRACE drought indicators. By applying three methods (GRACE-based indicators), the authors assessed the skills of newly derived GRACE drought indicators under rather more controlled conditions. This work is significant, as the study is a considerable addition to the existing literature about drought identification methods. Also, the topic is within the scope of Hydrology and Earth System Sciences. Overall, the experimental design is clear, and for the most part, the authors' conclusions are supported by their 
findings. However, I outline several general concerns, followed by a range of specific comments, which prevent me from recommending this manuscript for publication in its current form. I do hope though that the authors will be able to adequately address my comments and when that is done, this paper should be acceptable for publication.

1. The paper is relatively poorly written. There is a significant number of grammatical/syntactic errors that are present throughout the entire body of the manuscript. I specify several of these in the "Specific Comments" section below, but the authors need to thoroughly check the entire text, as similar or other mistakes may exist elsewhere. 2. Page 3 Line 14 "As can be expected, TWSC and 6 months SPI appear moderately similar (correlation 0.43 ), characterised by positive peaks e.g. at the beginning of 2004 and at the end of 2009, and negative peaks at the beginning of 2013. This motivates us to modify common GRACE indicators. .." I find the evidence not supportive enough to safely conclude that this link/association between TWSC and SPI is always (or everywhere) the case. The authors should test this on several different regions characterized by varying hydro-climatic conditions. Making such conclusive statements using only one example is scientifically inaccurate. 3 . More information is required for the cluster identification. How exactly were the three clusters determined? The authors also need to clearly specify their exact geographic locations. 4. The authors should provide more detailed information (characteristics) about specific droughts mentioned in their methodology section.

Specific Comments-

\section{Abstract}

"Thus, this study aims at a better understanding of how drought signals, in the presence of trends and GRACE-specific spatial noise, propagate through GRACE drought indicators": This phrase is perhaps the essence of the abstract; therefore it should be able to provide the necessary information on its own. The authors need to specify which trends they are referring to. Line 10 application-dependent Line 10 large differ-

Printer-friendly version

Discussion paper 
ences Line 11 particularly Line 12 We show that trends and accelerations - what do the authors mean by "accelerations"?

Page 1 Line 17 affect the Line 18 replace "reach" with "range" Line 24 led

Page 2 Line 4 depends on the accumulation period considered - unclear Line 16 Much fewer Line 23 and the first data are expected Line 27 they found good agreement to net precipitation minus evaporation. - unclear Line 34 without utilizing external information - please specify

Page 3 Line 4 delete "e.g." Line 7 "smoothing" Line 17 What are "differencing periods"? Line 21 spatially averaged Line 26 will complete the paper

Page 4 Line 2 explore Line 10 more regularly

Page 8 Line 10 we construct Line 13 including the introduced (in Sec. 2.3) signal ... Line 26 ... following $A$ et al. (2013).....is there something missing here?

Page 11 Line 8 drought onset and end Lines 10-14 these thresholds are rather arbitrarily made. It seems to me that a single value for the drought duration and magnitude should not be used for different hydrologic regimes.

Page 12 Line 5 inappropriate use of English for a scientific paper

Page 13 Line 10 delete "would"

Page 14 Line 17 for the 3 , and 6 months differenced DSID

Page 20 Line 24 climatic phenomenon Line 24 delete "related to climatic conditions" as it is redundant

Page 21 Line 9 in the northeastern

Printer-friendly version

Page 23 Line 22 particularly Line 25 the onset and end

Discussion paper

Please also note the supplement to this comment: 
https://www.hydrol-earth-syst-sci-discuss.net/hess-2019-268/hess-2019-268-RC2supplement.pdf

Interactive comment on Hydrol. Earth Syst. Sci. Discuss., https://doi.org/10.5194/hess-2019268, 2019. 